\title{
Change of practice key to improving patients' access to psychiatrists
}

$\mathrm{W}$ ith one in five Canadians expected to experience a mental illness in their lifetime and a dwindling number of psychiatrists, is the supply of mental health services on track to meet demand?

The quick answer is probably not. The more complex answer is that this is the wrong question.

"There will never be enough psychiatrists to address all the mental health needs of Canadians - nor should there be," says Dr. David Goldbloom, chair of the Mental Health Commission of Canada. While the number of psychiatrists may be one aspect to the access problem, Goldbloom thinks this argument has gone stale.

The key to addressing problems with access to mental health services in Canada is to change how psychiatrists practise. But until we do so, Goldbloom is quick to acknowledge that "We're currently not doing too well."

According to the National Physician Survey, the average wait time for nonurgent cases across Canada in 2010 was 11 weeks in urban areas and up to 20 weeks in rural areas. In addition, only $16 \%$ of urgent patients were seen within the day. Not surprisingly, 55\% of family physicians rated access to psychiatric care as fair or poor.

The underlying reasons are twofold.

Firstly, the specialty is suffering from aging pains, according to a report from the Canadian Medical Association (CMA). Psychiatry has a disproportionately large number of older physicians compared to most other specialties: $51 \%$ are age 55 or older, while the norm in other specialties is around $40 \%$. Not surprisingly these older psychiatrists are planning to retire, and when that happens, over the next five years, it will mean a loss of about 350 of the 4426 practicing psychiatrists - some $8 \%$.

Secondly, recruiting residents has been problematic for a long time. Rea-

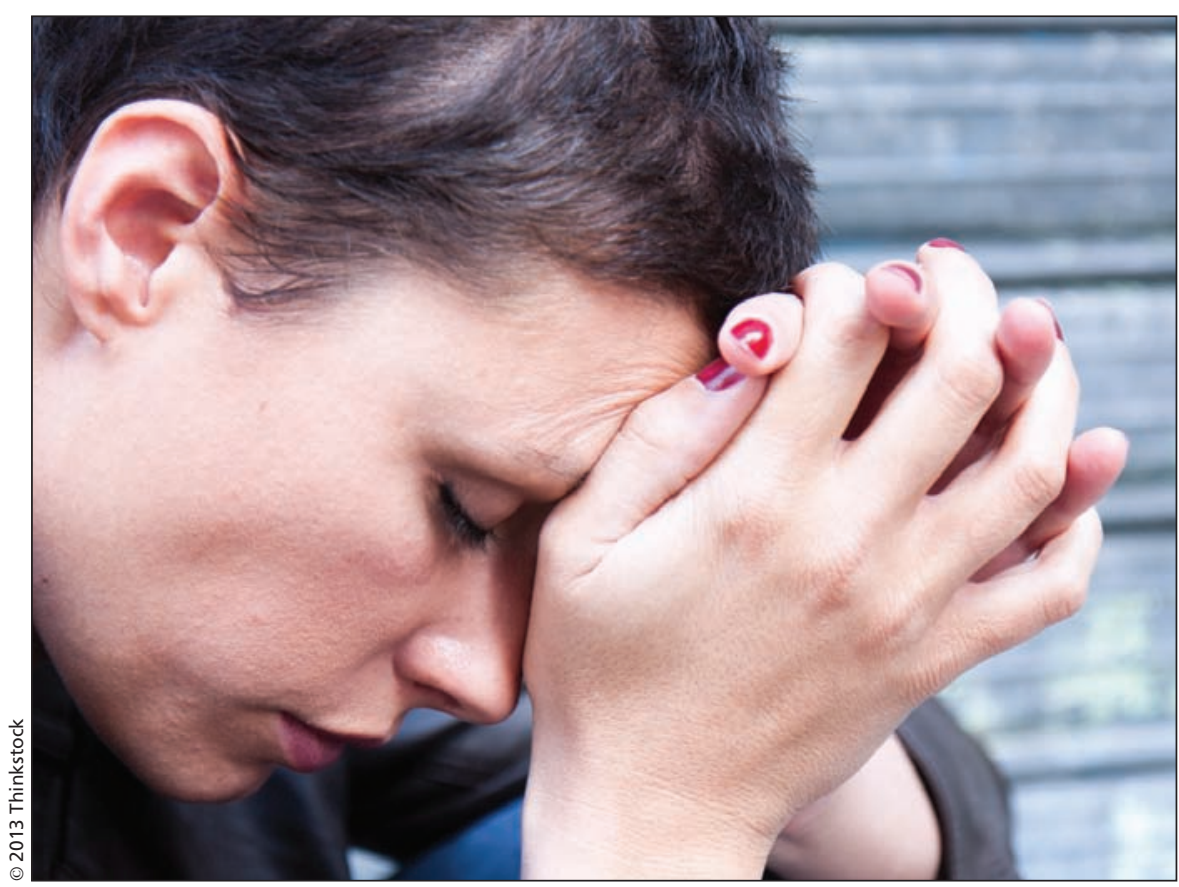

The average wait time for nonurgent psychiatric cases across Canada in 2010 was 11 weeks.

sons include difficulties with patients, lack of scientific foundation, poor role models, and perceived low status, prestige, and financial reward (Acad Psychiatry 2013;37:150-7).

There are some exceptions. Dr. Jodi Lofchy, director of Psychiatry Emergency Services at the University Health Network in Toronto, Ontario, created The Psychiatry Institute for Medical Students back in 1994 to give first- and second-year medical students more early experience with psychiatry, including access to role models, mentors and potential elective supervisors. The strategy has worked. Since 2008, the University of Toronto has regularly filled all 30 of its psychiatric residency positions - the largest psychiatry residency program in North America. Lofchy thinks this recruitment strategy should be adopted by other medical schools (Acad Psychiatry 2010;34:150-3).

For now, however, the University of Toronto's success is unique. Official results of the 2013 Canadian Resident Matching Service are yet to be released, but the 2012 results indicate 18 psychiatry residency spots $(\sim 13 \%$ of 124) went vacant - this has been the case for the past five years.

An aging specialty and recruitment issues mean that the number of psychiatrists per 100000 people is projected to fall from approximately 13.9 in 2010 to 11.9 by 2030 , according to the CMA. The Canadian Psychiatric Association states that 15.2 psychiatrists are needed per 100000 population.

At the Mental Health Commission of Canada, Goldbloom doesn't think the answer lies solely in numbers.

He points to the City of Toronto, which has 60 psychiatrists per 100000 people; far more than the national average of around 14. "One would conclude that access to a psychiatrist is easy is Toronto," he says. "But that unfortunately is just not the case. Arguments about the number of 
psychiatrists are therefore somewhat stale. We should be asking not simply how many psychiatrists there are but what they do with their professional time."

Goldbloom and the board of the Mental Health Commission of Canada, which was created in 2007 , are working on a number of strategies to increase accessibility to mental health care in Canada.

These include adjusting incentives and fee schedules, so that the limited number of psychiatrists are used more efficiently. For example, Goldbloom suggests that regional disparities in access to psychiatric care may be helped by the use of technologies such as televideo conferencing and Skype.

Other solutions include adjusting fee schedules to provide an incentive for psychiatrists to take on more complex cases, while encouraging them to share care of less complex cases with multidisciplinary teams. These teams include both primary care physicians and nonphysician mental health professionals, such as social workers, occupational therapists, counselors and peer support workers.

Some psychiatrists, including Dr. Daniel J. Carlat in his book Unhinged: The Trouble With Psychiatry - A Doctor's Revelations About a Profession in Crisis, say the strategy of encouraging psychiatrists to see only the most complicated cases may adversely affect practice, causing causing psychiatrists to become "pill pushers."

But Goldbloom strongly disagrees.

"Psychotherapy can be a very effective treatment modality, even for the most complicated patients - there is no reason to think that psychiatrists will end up pill pushing."

The Mental Health Commission of Canada is beginning to implement many of these strategies as well as other initiatives they outlined in Canada's first mental health strategy, published in May 2012.

For example, the mental health and addictions services in Regional Health Authorities across Saskatchewan have been using a novel, "Lean" approach to reduce wait times. Part of the Lean approach involves evaluating incentives for necessary services in order to make processes and procedures more efficient and more responsive. One of the larger health authorities has reduced wait times for adult mental health services from eight months to one month, and is meeting $80 \%$ of urgent requests within seven days. A smaller authority now has no waiting list for child and youth mental health and addictions services, and has reduced the waiting list for adult services by half.

In another example of using technology and multidisciplinary teams to help improve access to psychiatric care is the mental health commissionbacked pilot project, Strongest Families. Families receive handbooks and skill-demonstration videos, and work through step-by-step modules at home, supported by telephone, televideo and Skype consultations with trained coaches and other nonphysician mental health professionals. Using this novel strategy, the treatment drop-out rate was found to be less than $10 \%$, and children in the Strongest Families program were significantly less likely to still have a diagnosable illness after eight and 12 months (J Am Acad Child Adolesc Psychiatry 2011;50:1162-72).

Importantly, the efficacy of all the Mental Health Commission of Canada strategies is being tracked every five years using various indicators. Some examples include measuring the increase in the proportion of people with mood or anxiety disorders in the past year who consult a mental health professional, and the decrease in hospital readmission rates for mental illness within seven and 30 days.

In addition to the mental health commission's strategies, Lofchy and Goldbloom are both optimistic about the new residents starting their training, and think they are headed in the right direction with improving access to psychiatric care. "For the first time many psychiatry residents are taking the lead in implementing strategies to improve access to mental health care, and have increasingly become involved in more research on how psychiatrists manage their practice. Many residents are enthusiastic about the use of technology to improve access to care," says Goldbloom

Lofchy encourages psychiatry residents to go beyond their day-to-day clinical work. "New residents are increasingly taking on more social responsibility, expressing more willingness to collaborate in multidisciplinary teams, and the majority now express a strong recognition for their role as advocates of mental health." - Paul Kudlow MD, Toronto, Ont.

CMAJ 2013. DOI:10.1503/cmaj.109-4574 